\title{
First fruits
}

In 2004, the Molecular Libraries Initiative, a programme through which the US National Institutes of Health (NIH) provides academic researchers with access to high-throughput screening (HTS) technologies and expertise, was started. A success arising from the first assay to be accepted for screening by this initiative - the identification of a novel class of lead compounds for schistosomiasis - has now been reported in Nature Medicine.

Schistosomiasis (also known as bilharzia and snail fever) is a chronic, parasitic disease caused by flatworms of the genus Schistosoma. New treatments for this neglected tropical disease are badly needed as current therapy relies almost exclusively on the drug praziquantel, raising concern about the possible emergence of resistance.

Inside their mammalian hosts, the schistosome parasites survive in an aerobic environment and, therefore, need to minimize damage from reactive oxygen species generated both by their own aerobic respiration and by the host's immune system. This protection is afforded by a single selenocysteine-containing flavoenzyme, thioredoxin glutathione reductase (TGR). Previous studies have shown that TGR is an attractive potential drug target for the treatment of schistosomiasis: it is essential for parasite survival and biochemically distinct from equivalent enzymes in vertebrates (glutathione reductase and thioredoxin reductase).
In order to identify new drug leads that target TGR, David Williams and colleagues at Illinois State University collaborated with the NIH Chemical Genomics Center to carry out a quantitative high-throughput screen of the Molecular Libraries Small Molecule Repository. They found that phosphinic amides and oxadiazole 2-oxides were effective inhibitors of TGR and thus, potential new drug leads for schistosomiasis therapy.

The authors subsequently focused their analyses on two compounds (one of each type). A phosphinic amide and an oxadiazole 2-oxide inhibited TGR in vitro in a time-dependent manner at nanomolar and low micromolar concentrations, respectively. Furthermore, with cultured parasites, $100 \%$ worm mortality was recorded at concentrations that are well tolerated by mouse myeloma cells.

The oxadiazole 2-oxide was active against all three major schistosome species infecting humans, and its effects were at least in part dependent on its nitric oxide production, as simultaneous treatment with a nitric oxide scavenger extended worm viability. Importantly, decreases in worm burdens of at least $88 \%$ were observed at all stages of infection when $10 \mathrm{mg}$ per $\mathrm{kg}$ of the oxadiazole 2-oxide was administered by intraperitoneal injection once a day for 5 days to S. mansoni-infected mice 1, 23 and 37 days after infection. The oxadiazole 2-oxide was also shown to significantly reduce parasite-egg-induced

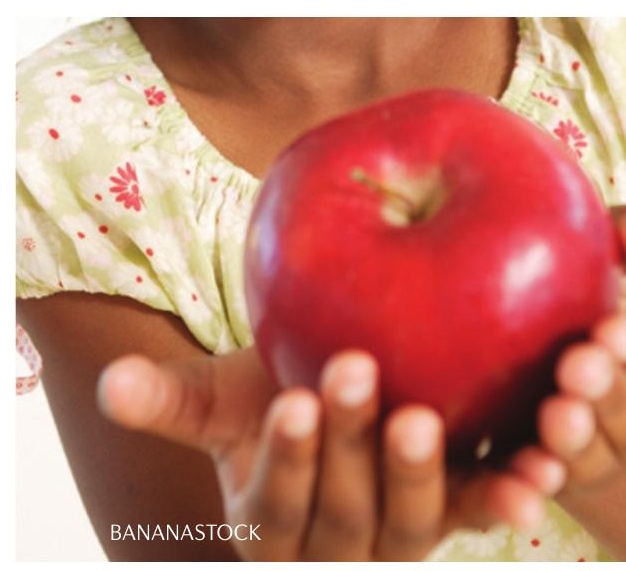

pathologies. In particular, the effects of this compound surpassed the criteria set by the World Health Organization for lead compound development.

Together, these findings show that oxadiazole 2-oxides that target parasite TGR represent new lead compounds for the development of an alternative schistosomiasis therapy that might help prevent the emergence of praziquantel-resistant strains. Moreover, this study illustrates how research to combat neglected diseases of the developing world can be aided by teaming academic investigators knowledgeable about the pathogens with investigators in centres with assay, HTS and chemical optimization expertise.

Monica Hoyos Flight

ORIGINAL RESEARCH PAPER Sayed, A. A. et al. Identification of oxadiazoles as new drug leads for the control of schistosomiasis. Nature Med. 16 March 2008 (doi:10.1038/nm1737) FURTHER READING Kuntz, A. N. et al. Thioredoxin glutathione reductase from Schistosoma mansoni: an essential parasite enzyme and a key drug target. PLoS Med. 4, e206 (2007) | Simeonov, A. et al. Quantitative high-throughput screen identifies inhibitors of the Schistosoma mansoni redox cascade. PLoS Negl. Trop. Dis. 2 e127 (2008) 